Rev. Adm. Saúde (On-line), São Paulo, v. 20, n. 81: e216, out. - dez. 2020, Epub 27 dez. 2020 http://dx.doi.org/10.23973/ras.81.216

ARTIGO ORIGINAL

\title{
Desafio na gestão hospitalar: diferenças entre o planejamento de consumo e o consumo efetivo de medicamentos
}

Challenge in hospital management: differences between consumption planning and effective medication consumption

Gídeon Depintor Duarte', Beatriz Aparecida Ozello Gutierrez², Rosana Alves Vieira ${ }^{3}$, Teresa Mota Pereira ${ }^{4}$, Thais Bento Lima-Siva ${ }^{5}$

1. Graduando em gerontologia da Escola de Artes, Ciências e Humanidades da Universidade de São Paulo (EACH-USP), São Paulo SP.

2. Docente do Curso de Graduação em Gerontologia da EACH-USP, São Paulo SP.

3. Servidora do Departamento Administrativo do Hospital Universitário da Universidade de São Paulo (HU-USP), São Paulo SP.

4. Servidora do Departamento Administrativo do HU-USP, São Paulo SP.

5. Docente do Curso de Graduação em Gerontologia da EACH-USP, São Paulo SP

\section{RESUMO}

Estima-se que de 35 a $45 \%$ do orçamento total do hospital é associado à administração de materiais, sendo a gestão de estoque um processo importante na gestão estratégica voltada para a otimização de gastos em saúde. Assim, a gestão de estoque é desafiadora, pois o seu controle demanda a melhoria contínua dos seus processos. O presente estudo objetivou analisar os processos de gestão de medicamentos em hospital universitário. Essa pesquisa quantitativa de caráter transversal e exploratório desenvolvida em hospital universitário do município de São Paulo, Brasil, analisou a previsão de consumo dos medicamentos, elaborada pelo setor do almoxarifado (excluindo- 
se soros e químicos), comparando com o consumo declarado pelas unidades. Os dados encontrados apontaram que existe a oportunidade de melhora no fluxo logístico em algumas unidades áreas em que a previsão de consumo é significativamente maior que o consumo efetivo e, por outro lado a previsão é insuficiente para atender a demanda em uma das unidades. Contrapondo-se a esses desajustes, identificou-se que a previsão de consumo e consumo efetivo declarado estão equiparadas em outras unidades. A utilização do sistema de gerenciamento de materiais e a gestão dos setores envolvidos permitem que o hospital tenha êxito no planejamento demonstrado nas previsões de consumo que são equivalentes ao consumo efetivo.

Palavras-chave: Acesso a Medicamentos Essenciais e Tecnologias em Saúde, Administração de Serviços de Saúde, Hospitais Universitários.

\section{ABSTRACT}

It is estimated that 35 to $45 \%$ of the hospital's total budget is associated with materials management, with inventory management being an important process in strategic management aimed at optimizing health spending. Thus, inventory management is challenging, as its control demands the continuous improvement of its processes. The present study aimed to analyze the medication management processes in a university hospital. This cross-sectional and exploratory quantitative research carried out at a university hospital in the city of São Paulo, Brazil, analyzed the forecast for the consumption of medicines, prepared by the warehouse sector (excluding serums and chemicals), comparing with the consumption declared by the units. The data found pointed out that there is an opportunity for improvement in the logistical flow in some units in which the consumption forecast is significantly higher than the actual consumption and, on the other hand, the forecast is insufficient to meet the demand in one of the units. In contrast to these imbalances, it was identified that the consumption forecast and declared effective consumption are similar in other units. The use of the materials management system and the management of the sectors involved allow the hospital to be successful in the planning shown in the consumption forecasts that are equivalent to the actual consumption.

Keywords: Access to Essential Medicines and Health Technologies, Health Services Administration, Hospitals University.

\section{INTRODUÇÃO}

O aumento dos gastos em saúde no Brasil e no mundo, tem sido vinculado ao processo de envelhecimento demográfico e à transição de novas tecnologias 
para o tratamento em saúde, destaca-se na literatura que os recursos direcionados ao sistema de saúde são limitados ${ }^{1}$.

No Brasil em média, se tem uma abrangência de quase $80 \%$ nos casos de atendimento médico de emergência, que é responsável por um quarto das consultas ambulatoriais realizadas nos serviços hospitalares, principalmente os vinculados ao Sistema Único de Saúde (SUS), sendo uma referência para a população no que se relaciona ao atendimento em saúde².

A maioria dos hospitais universitários (HU's) é considerada como sendo organização de média ou alta complexidade, em nosso país, predomina o vínculo à gestão pública, deste modo necessitam de eficácia no planejamento e nos processos de gestão ${ }^{2,3}$. Essas instituições apresentam foco na eficiência de suas atividades, assim como em uma maior satisfação do usuário em complemento ao sucesso das intervenções técnicas esperadas no âmbito do tratamento e prevenção de danos à saúde ${ }^{3}$.

Estima-se que 35 a $45 \%$ do orçamento total do hospital é associado à administração de materiais sendo a gestão de estoque um processo importante para o alcance da gestão estratégica voltada para a otimização de custos em saúde ${ }^{4}$. Neste contexto, a gestão de estoque é uma gestão desafiadora, pois o seu controle demanda a melhoria contínua dos seus processos, exigindo ferramentas apropriadas para o levantamento de indicadores de qualidade, reduzindo assim seus gastos e proporcionando uma assistência ao paciente com mais segurança e qualidade ${ }^{4}$.

A gestão eficiente de materiais médico-hospitalares necessita que os profissionais envolvidos nos processos de compra, estocagem e distribuição desses materiais sejam capacitados visando evitar o desabastecimento ou desperdício ${ }^{5}$. Facchini 6 destacou que administrar os estoques de instituições hospitalares é garantir o atendimento das demandas atuais e controlar os investimentos para demandas futuras, equilibrando a importância de atendimento ao cliente com as necessidades de minimizar os investimentos financeiros, sendo uma função-chave na gestão em saúde.

No contexto dos HU's sabe-se que essas instituições enfrentam uma realidade, em que precisam otimizar seus processos, reduzindo custos operacionais a fim de proporcionar uma alocação mais eficiente de seus recursos, pois o financiamento público dispõe uma quantidade limitada de recursos. Em contraponto ao aumento da demanda da exigência em relação à qualidade do serviço ${ }^{7}$.

A restrição orçamentária exerce significativa importância na gestão hospitalar, pois prioriza a redução do custo de aquisição de produtos, a busca por parceiros socialmente responsáveis, a valorização da segurança e da confiança, somado à modernização de suas estruturas física, humana e administrativa ${ }^{8}$.

Nessa abordagem, vale destacar que a logística de materiais é um ponto importante dentro da gestão de HU's de financiamento público, uma vez que trata da otimização dos fluxos gerenciais, nas mais diversas áreas e setores, 
objetivando a eficiência operacional. Assim, o almoxarifado é um desses setores que merece o foco da gestão em serviços de saúde, devido à sua importância relacionada ao controle e à manutenção de estoques, equilibrando as oscilações inesperadas da demanda ${ }^{9}$.

Partindo-se desta premissa, buscou-se desenvolver, dentro da divisão administrativa de HU, a categorização de dados sobre os medicamentos referentes a seis setores médicos. O presente estudo objetivou analisar os processos de gestão de medicamentos em hospital universitário. Essa pesquisa quantitativa de caráter transversal e exploratório desenvolvida em hospital universitário do município de São Paulo, Brasil, analisou a previsão de consumo dos medicamentos, elaborada pelo setor do almoxarifado (excluindose soros e químicos), comparando com o consumo declarado pelas unidades.

\section{MÉTODO}

Trata-se de estudo quantitativo, transversal, exploratório e descritivo.

O estudo foi desenvolvido em um HU de nível secundário localizado no município de São Paulo, Brasil, que presta assistência em saúde, realiza pesquisa, e formação profissional e, atualmente conta com 280 leitos ativados.

Foram analisadas as previsões de consumo dos medicamentos, elaboradas pelo setor do almoxarifado (excluindo-se soros e químicos), comparando com o consumo declarado pelas áreas de Alojamento Conjunto, Berçário, Clínica Médica, Clínica Cirúrgica, Programa de Atendimento Domiciliar, Pediatria, Unidade de Terapia Intensiva Adulto - UTI Adulto - (UTI Adulto), Unidade de Terapia Intensiva Adulto - (UTI Pediátrica), Unidade de Terapia Intensiva Pediátrica - (UTI Pediátrica) e pelo projeto Estudo Longitudinal de Saúde do Adulto (ELSA).

Os dados foram extraídos do software do hospital no Sistema de Gestão de Materiais (SGM), trata-se de programa interno da instituição, referente ao período de outubro de 2018 a março de 2019.

A escolha das variáveis direcionou-se na demanda do HU em monitorar indicadores de desempenho operacional que permitissem otimizar os recursos do setor de almoxarifado. Nesse sentido, realizou-se uma análise descritiva e comparativa, do consumo previsto de medicamentos pelo planejamento administrativo, comparado ao consumo efetivo das unidades elencadas do HU e o potencial impacto no planejamento do setor de Almoxarifado no que se refere aos medicamentos.

A elaboração dos indicadores iniciou-se com o recebimento da previsão de consumo no período de seis meses disponibilizado pelo setor de almoxarifado no hospital. Estes dados foram codificados de forma a apresentar o consumo mensal de cada medicamento.

O programa estatístico utilizado foi o Excel v. 2013 e posteriormente, foram elaborados gráficos descritivos. Realizou-se a comparação das médias dos 
seis meses analisados com a quantidade informada nas etapas de planejamento e consumo.

Foram utilizadas variáveis quantitativas referentes ao consumo de medicamentos, excluindo-se os soros e os químicos, divididas em categorias e comparadas entre consumo previsto e efetivo dos medicamentos com maior volume de uso em cada uma das áreas analisadas. Os dados foram extraídos do Sistema interno de Gestão de Materiais - (GSM).

\section{RESULTADOS}

O presente estudo objetivou-se realizar análises descritivas e comparativas da previsão de consumo de medicamentos elaborada pelo setor de almoxarifado e pelo consumo efetivo declarado pelas áreas supridas com o objetivo de visando verificar se os indicadores despesas são convergentes ou divergentes.

\section{Estimativa de consumo}

Na Figura 1 observa-se as previsões de medicamentos distribuídos nas unidades do HU.

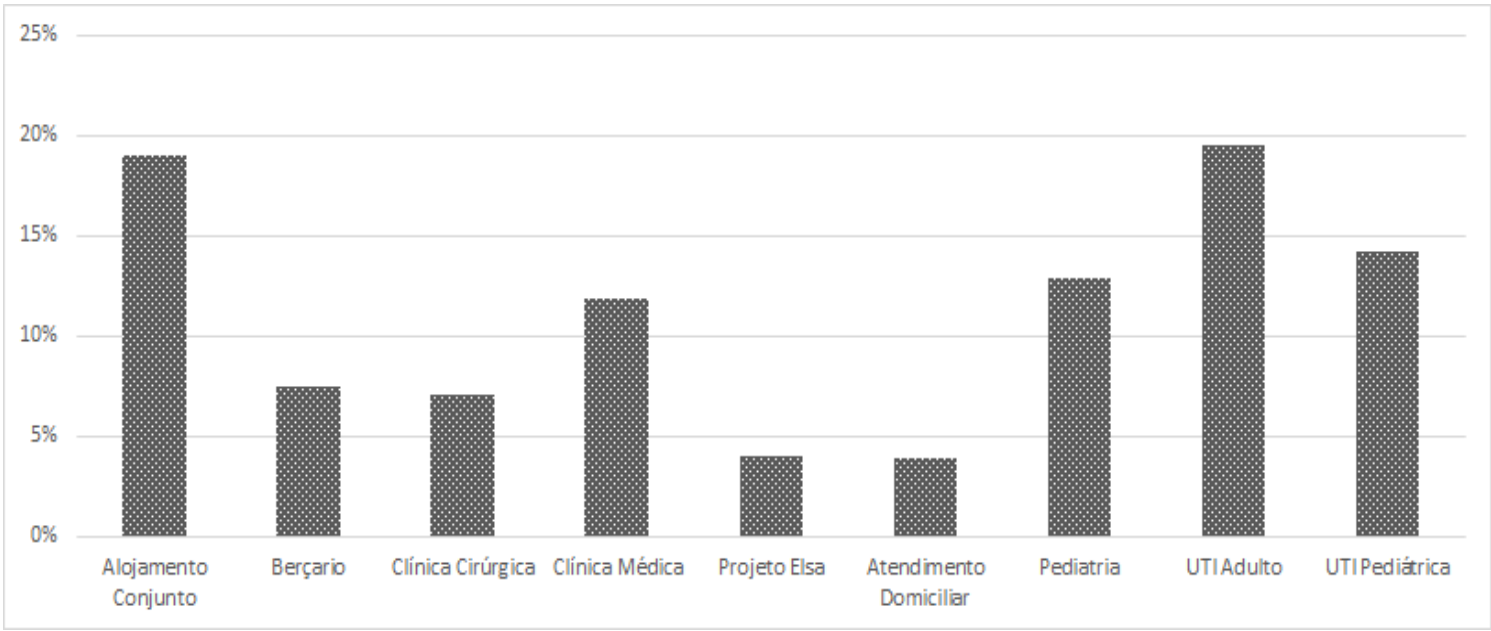

Figura 1. Distribuição dos medicamentos em cota para cada unidade do HU, 2019.

Conforme Figura 1 a UTI Adulto foi a unidade que obteve maior previsão de medicamentos. 
A Figura 2 apresenta o dado comparativo entre a previsão de consumo elaborada pelo planejamento no setor do almoxarifado e o que efetivamente foi dada baixa de consumo nas unidades do HU.

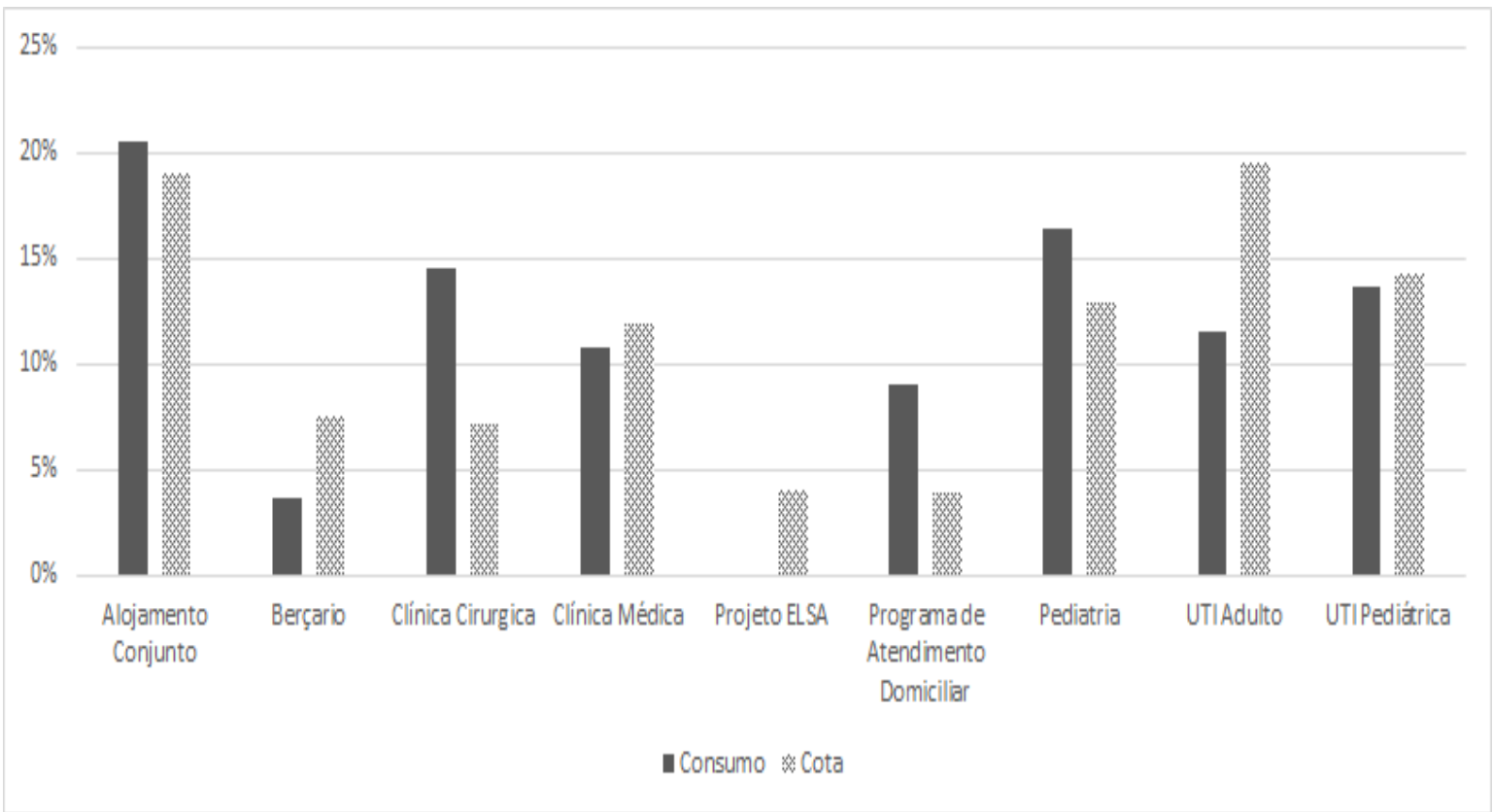

Figura 2. Dados comparativos entre previsão de consumo e consumo efetivo, 2019.

A partir da Figura 2 verifica-se que a UTI Adulto foi a unidade que apresentou maior divergência entre consumo efetivo e previsão de consumo. Nos dados relacionados ao item específico, a cota prevê que apenas cinco medicamentos (cloreto de sódio a $0,9 \%$, água destilada, papaína pó, cloreto de sódio a $20 \%$ e cloreto de potássio a $19,1 \%$ ) são responsáveis pelo total de $50 \%$ das dispensas no período analisado. Enquanto 376 itens são responsáveis pelos outros 50\%, nomeado como demais.

Em relação à distribuição do grupo de medicamentos mais consumidos verificase na Figura 4 que o maior consumo destes itens ocorre na Pediatria.

\section{Consumo Efetivo}

O consumo efetivo dos medicamentos consiste no procedimento de baixa, do uso de determinado item. Nestes dados não foram considerados medicamentos de consumo os itens que não apresentaram procedimento de baixa no Sistema de Gestão de Materiais (GSM). 
Por meio da Figura 3 observa-se a distribuição dos medicamentos de consumo efetivo por unidade do HU.

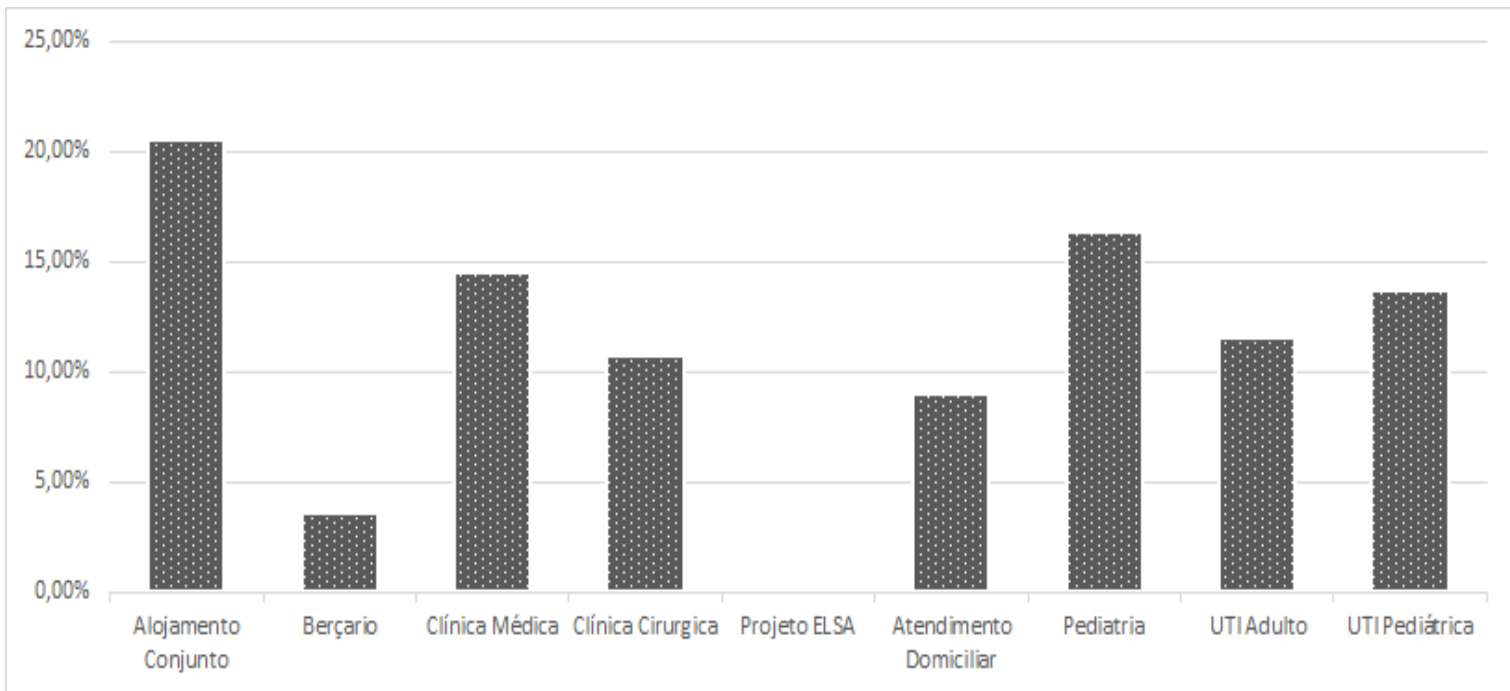

Figura 3. Distribuição dos medicamentos com consumo efetivo declarado pelas unidades do HU, São Paulo, 2019.

Nota-se que o setor Alojamento Conjunto possui o maior volume consumido e os setores Pediatria e UTIs Adulto e Pediátrica representam aproximadamente $40 \%$.

A Figura 4 apresenta a proporção dos medicamentos com maior volume no consumo efetivo declarado pelas unidades do HU.

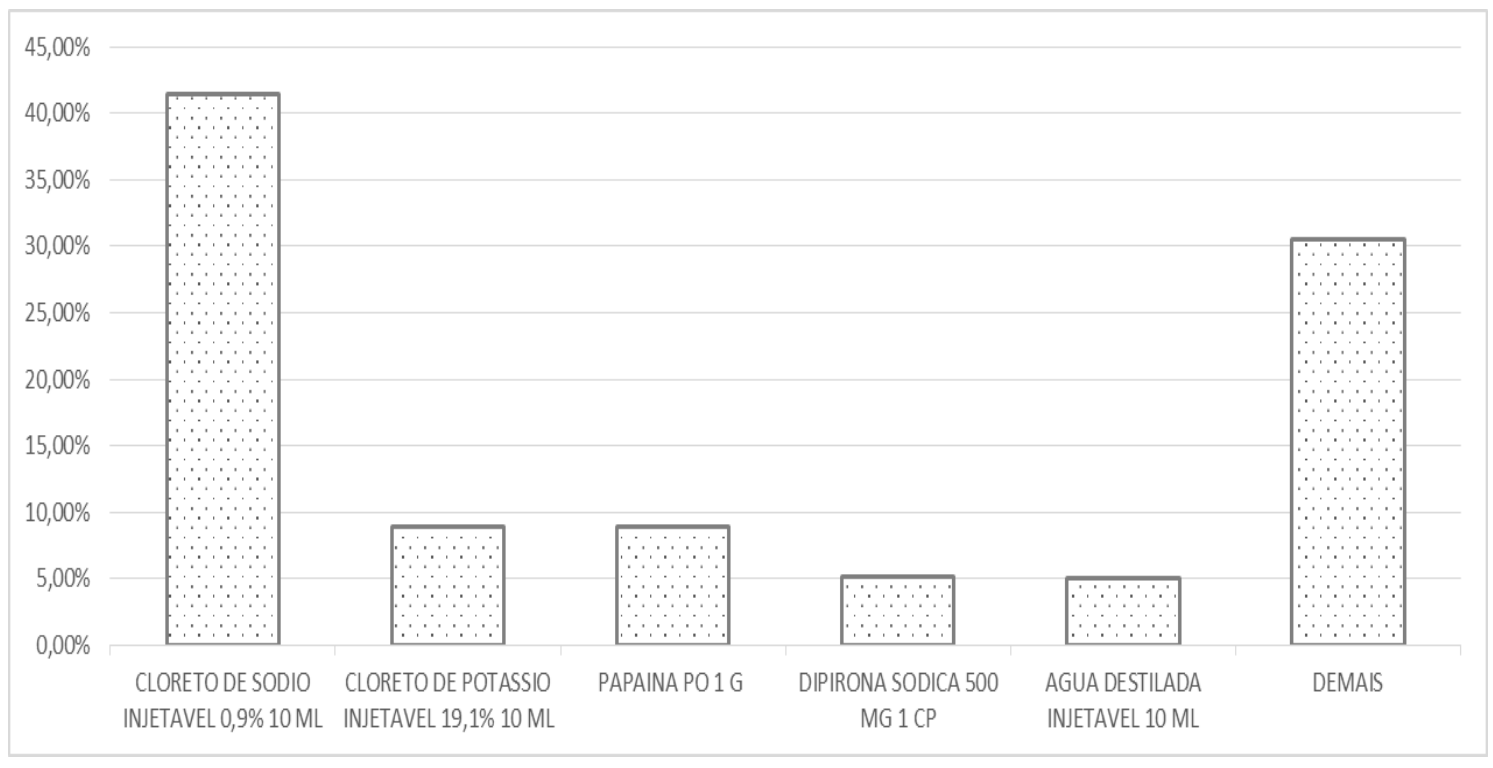


Figura 4. Proporção dos medicamentos com maior volume no consumo efetivo declarado, São Paulo, 2019.

Em relação à variável consumo efetivo declarado pelos setores analisados, observa-se que estes itens apresentam o mesmo padrão de consumo previsto na cota, em que $50 \%$ corresponde a cinco medicamentos conforme Figura 5.

Na Figura 5 estão distribuídos os cinco medicamentos com maior previsão de consumo nas unidades do HU.

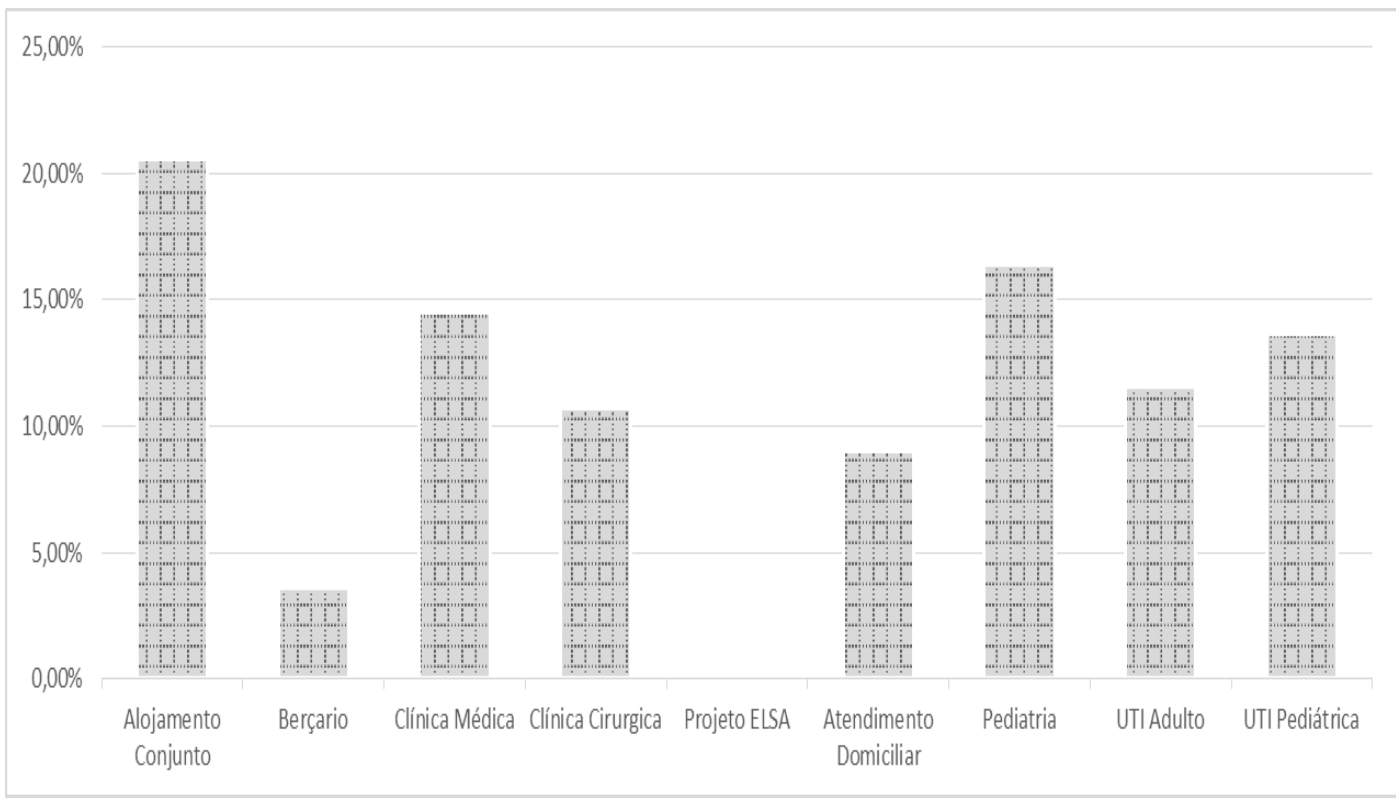

Figura 5. Distribuição dos cinco medicamentos com maior previsão de consumo nas unidades do HU, São Paulo, 2019.

De acordo com a Figura 6, verifica-se que as unidades de Pediatria e UTIs Adultos e Pediátrica consomem $50 \%$ dos cinco itens com maior consumo efetivo dentre as unidades analisadas.

A partir da Figura 6 pode-se ver o comparativo da distribuição dos medicamentos em previstos e consumidos nas unidades do HU. 


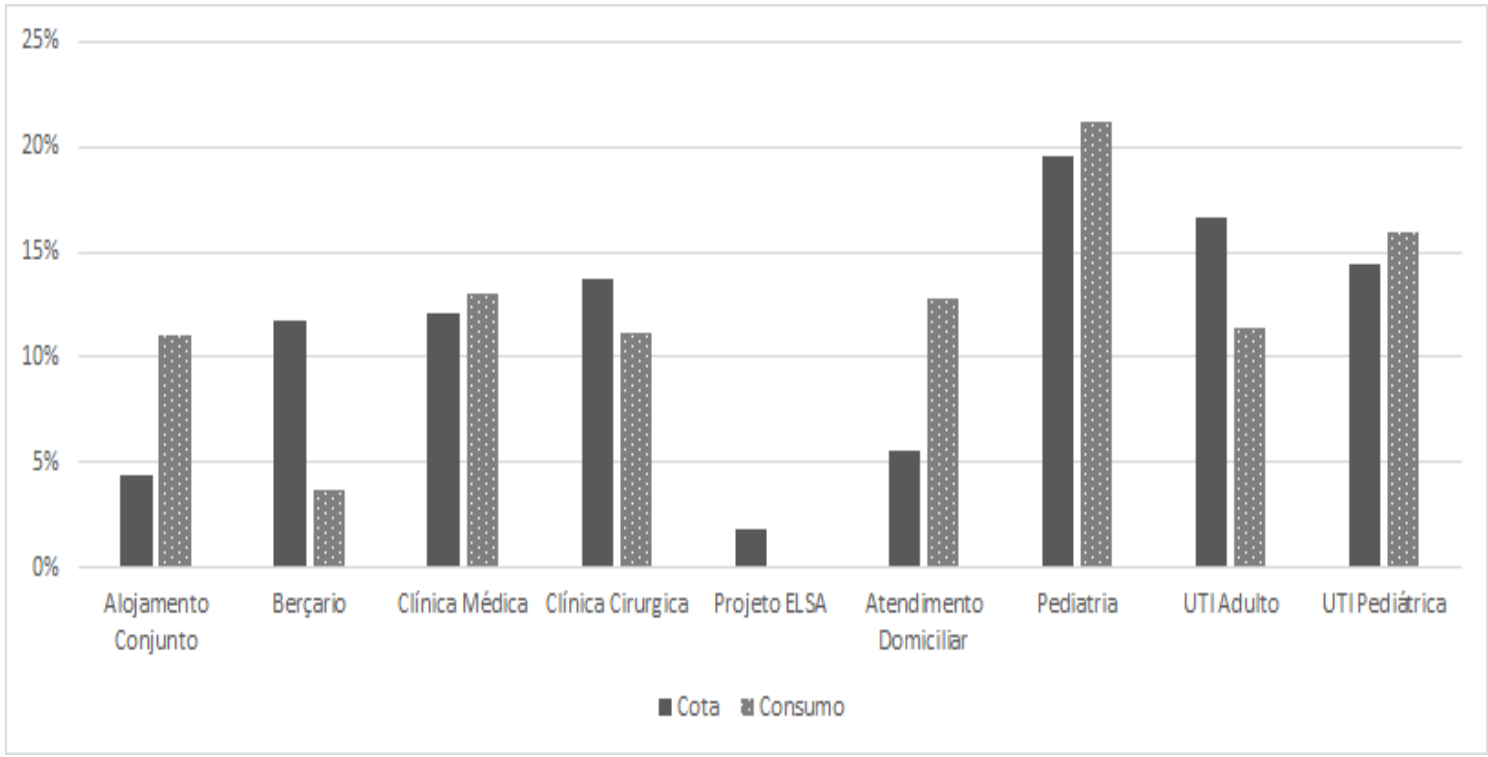

Figura 6. Comparativo da distribuição dos medicamentos em previstos e consumidos nas unidades do HU, São Paulo, 2019.

O comparativo proporcional entre a cota e o consumo efetivo presente na Figura 7 , mostra que a previsão de consumo é precisa no que se refere às unidades estudadas, exceto ao Alojamento Conjunto. Nesta unidade no qual o consumo é significativamente maior que o previsto e na UTI Adulto e Berçário prevê-se um consumo maior do que é efetivamente consumido. A Figura 8 aponta o comparativo da previsão e do consumo efetivo dos medicamentos com maior volume de consumo.

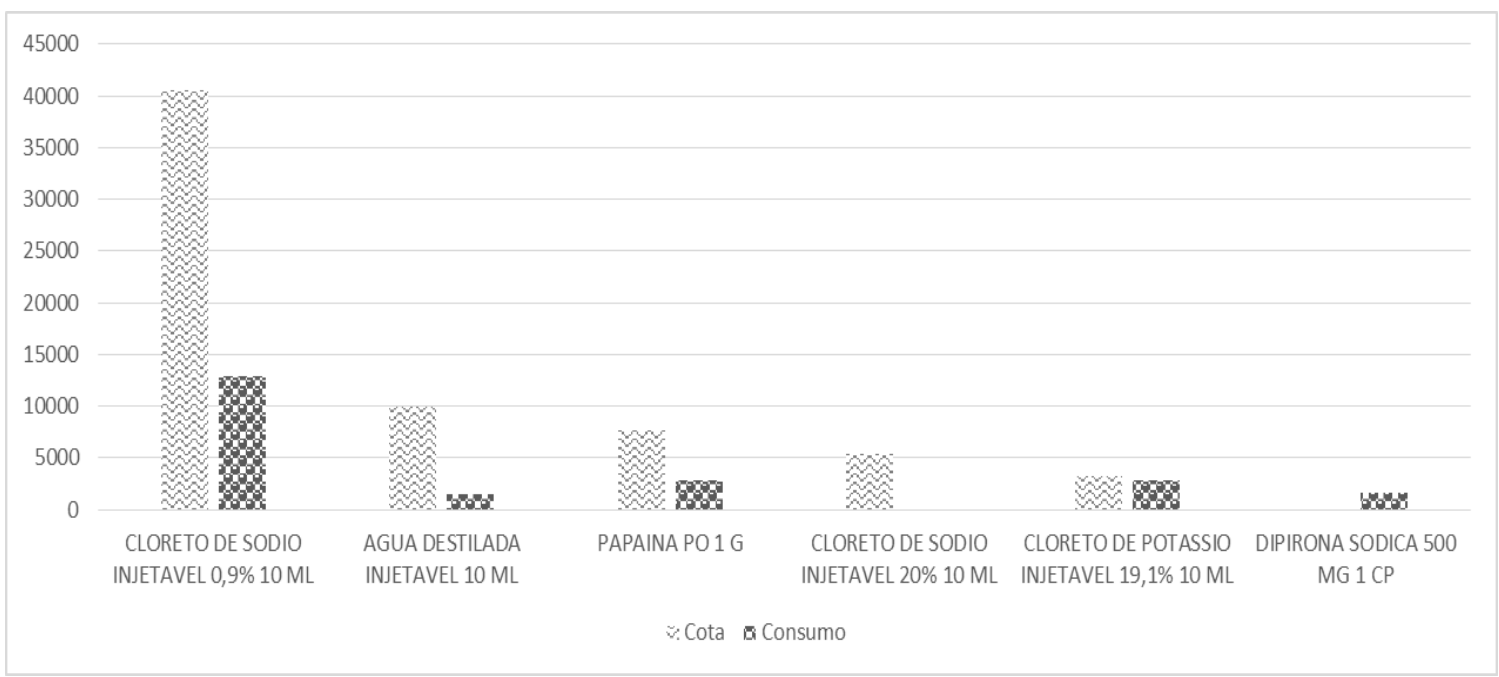

Figura 7. Comparativo da previsão e do consumo efetivo dos medicamentos com maior volume de consumo, São Paulo, 2019. 


\section{DISCUSSÃO}

Com o foco na prevenção, promoção de saúde e assistência, os sistemas públicos de saúde são responsáveis por atender grande parte desta da população. A restrição orçamentária, envelhecimento populacional, conjuntura político-econômica e complexidade de gestão deste seguimento tornam os modelos de gestão logística uma ferramenta importante para o dia a dia dos diversos serviços de saúde independente da complexidade da atenção ${ }^{10}$.

A logística de materiais é, sem dúvida, fundamental para os processos de gestão de qualquer empresa, independente do segmento. No que se refere aos serviços de saúde, a satisfação do usuário é primordial, em um cenário em que a rapidez e a eficiência na comunicação para aquisição, armazenagem e distribuição de materiais e medicamentos estão ligadas às condições de saúde, sendo parte indispensável dentro do planejamento estratégico de qualquer unidade de saúde independente do porte ou complexidade de atendimento ${ }^{11}$.

Com base neste contexto objetivou-se realizar um estudo para identificar pontos de potencial de aprimoramento no processo logístico, especificamente nas etapas previsão de consumo comparada à declaração de consumo nas unidades do HU. No presente estudo, os dados encontrados apontam que existe a oportunidade de melhora no fluxo logístico em algumas áreas em que a previsão de consumo é significativamente maior que o consumo efetivo declarado como na UTI Adulto, por outro lado a previsão insuficiente para atender à demanda no Atendimento Domiciliar.

Nesse estudo, também foi identificado que a previsão de consumo e consumo efetivo declarado estão equiparadas no Alojamento Conjunto, UTI Pediátrica e Clínica Médica. A utilização do sistema de gerenciamento de materiais e a gestão das unidades envolvidas permitem que o hospital tenha êxito no planejamento demonstrado nas previsões de consumo que são equivalentes ao consumo efetivo.

Outros estudos da literatura corroboram com os achados do presente estudo, como o estudo de Kamimura ${ }^{11}$, sobre logística em serviço de saúde Para superar estes desafios é necessária a integração do sistema logístico visando alcançar o equilíbrio entre eficiência e tempo de resposta da cadeia de suprimentos da saúde, por meio de monitoramento constante e pelo gerenciamento da cadeia de suprimentos com o auxílio de sistemas de informação integrados ao fluxo de materiais e investimentos em tecnologia e automação na área de logística ${ }^{11}$.

Silva ${ }^{12}$ em seu estudo, apontou sobre a necessidade de integração dos sistemas logísticos para alcançar o equilíbrio entre demanda e resposta da cadeia de suprimentos da saúde, por meio de monitoramento constante e pelo gerenciamento da cadeia de suprimentos com o auxílio de sistemas de informação integrados ao fluxo de materiais e investimentos em tecnologia e automação na área de logística.

No que se refere à gestão, a logística hospitalar objetiva a constante disponibilidade de recursos para atender às demandas dos pacientes usuários 
do serviço por meio do planejamento, compra, armazenagem e gerenciamento dos itens que compõem o estoque buscando minimizar os custos com a manutenção ${ }^{13}$.

Para que haja pronta entrega dos medicamentos e insumos às diversas áreas hospitalares são necessários estoques, pois os processos de suprimento e demanda nem sempre podem ser sincronizados devido à natureza do processo de demanda e suprimento onde as necessidades de um não são idênticas às do outro ${ }^{14}$. Os desafios encontrados na logística de materiais está nas etapas de recebimento e separação dos itens pois esta atividade requer treinamento específico e auxílio de sistemas de gestão de materiais que proporcionem maior controle e menos desperdício, além de limitações físicas pois estoques ocupam grande quantidade de espaço estrutural dentro de qualquer atividade $^{15}$.

A adoção de sistemas logísticos possibilita a elaboração de indicadores de desempenho que estão diretamente relacionados à qualidade da assistência prestada aos usuários dos serviços pois subsidiam a tomada de decisão e alocação de recursos dos gestores destes locais. Neste contexto, o almoxarifado pode ser considerado um setor crítico na gestão de uma unidade de saúde, uma vez que é o setor responsável pela armazenagem e conservação, adequando os espaços, onde permanecerá cada item aguardando a necessidade do seu uso conforme a política geral de estoques da instituição ${ }^{5}$.

O processo logístico de materiais no almoxarifado de serviços de saúde pode ser gerenciado através da elaboração de indicadores pois são ferramentas que permitem o gerenciamento destes setores pois possuem informações de como os processos estão se desenvolvendo por meio de informações métricas objetivas sendo partes de ferramentas para gestão de qualidade principalmente quando estamos tratando de fluxos, possibilitando a identificação de pontos de melhoria no processo ${ }^{16}$.

A realidade dos equipamentos de atenção à saúde, em particular de HU, não permite a implantação de estoque próximo "a zero" com "máximo" giro de medicamentos pois, a manutenção do estoque está diretamente ligada à demanda instável inerente à atividade, o que exige atenção e cuidado ${ }^{17}$. Podese inferir que as perspectivas financeiras e/ou econômicas visam mensurar se as ações da logística reversa proporcionam aumento de receitas e redução de custos. Ou seja, as empresas devem atender seus objetivos financeiros e, ao mesmo tempo, oferecer valor ao seu cliente, sua principal fonte de receita ${ }^{18}$.

Este estudo se propôs a verificar se poderia ter divergências entre o que o HU prevê de consumo de medicamentos comparando com o consumo efetivamente declarado pelas unidades da instituição. Estas informações permitem ao gestor encontrar ferramentas de otimização, para a gestão dos custos de insumos do hospital por meio de planejamento mais preciso ao elaborar os indicadores que possam permitir a identificação, de pontos de reavaliação no fluxo de dispensa e consumo dos medicamentos pelas áreas médicas do hospital. 
As limitações deste estudo estão relacionadas às especificidades de cada unidade do HU, pois a grande variedade de medicamentos dispensados, associados à oscilação na demanda por consumo dificultam a identificação da etapa do processo que é passível de otimização sendo necessário a continuidade de análises destes processos e a realização de novos estudos verificando outros insumos hospitalares.

\section{AGRADECIMENTOS}

Agradecemos aos estagiários da Graduação do Bacharelado em Gerontologia, que contribuiriam auxiliando nas etapas iniciais da codificação dos dados: Ana Lúcia Fusco, Ana Carolina Ventura, Beatriz Guedes, Caroline Folli, Julia Azevedo, Murilo Lino e Sandra Cardoso. Agradecemos também aos funcionários do setor de estoque e almoxarifado do Hospital Universitário da Universidade de São Paulo, pelo o apoio para a realização do estudo.

\section{REFERÊNCIAS}

1. Ramos MC de A, da Cruz LP, Kishima VC, Pollara WM, de Lira ACO, Couttolenc BF. Performance evaluation of hospitals that provide care in the public health system, Brazil. Rev Saude Publica. 2015; 49.

2. IBGE. Estatísticas da Saúde Assistência Médico-Sanitária. 39, Brasil, Ministério do Planejamento, Orçamento e Gestão. 2010. 1-63 p.

3. Farias DC, De Araujo FO. Gestão hospitalar no brasil: Revisão da literatura visando ao aprimoramento das práticas administrativas em hospitais. Cienc e Saude Coletiva. 2017; 22(6):1895-904.

4. Pereira PM, Selingardi R. Proposta de redução de estoque excedente de um complexo hospitalar de alta complexidade. Rev Adm em Saúde. 2018; 18(70).

5. Melo AB, Gomes BR dos S, Pinheiro B do SB, Martins LFJ, Palheta MG, Santos RSU, Souza TCF, Silva IM. A gestão de materiais médico-hospitalar em hospital público. Rev. G\&S [Internet]. 2016; [citado 27 de dezembro de 2020]; 7(1): 369-387. Disponível em:

https://periodicos.unb.br/index.php/rgs/article/view/3433

6. Facchini E. Curva ABC e Estoque de Segurança. South Am Dev Soc J. 2019; 5(13):73.

7. Silva LAD et al. Logística: evolução internacional e nacional. Revista de Administração e Negócios da Amazônia. 2016; 8(3): 386-406. 
8. Dorneles C, Costa F, Guarnieri P. Gestão da aquisição e dos estoques de medicamentos: estudo de caso no Hospital Universitário de Brasília (HUB), Revista Foco. Jun. 2018; 11(2):28-52.

9. Brasileiro DON. Almoxarifado de um Hospital Universitário Federal. 2017; 2:50-61.

10. Reis ADC, Oliveira RP, Castro ADC. Logística Hospitalar: Uma Síntese do Estado da Arte. Rev Gestão e Desenvolv. 2018; 15(1):205.

11. Kamimura QP, et al. Logística em serviço de saúde: estudo em um hospital público. Lat Am J Bus Manag [Internet]. 2015; 6(1):132-43. Available from: https://www.lajbm.com.br/index.php/journal/article/view/238

12. Silva UR. Análise do processo de uma farmácia de medicamentos especializados e aplicação do lean healthcare. 2015; 1(30):179. Available from: www.teses.usp.br/teses/disponiveis/96/96132/tde-02092015100645/pt-br.php

13. Falk JA, Francisco J, Filho R. Logística hospitalar: um estudo sobre as atividades do setor de almoxarifado em hospital púbico. Rev Adm da UFSM. 2009; 2(1):59-79.

14. Gianesi IGN, Biazzi JL de. Gestão estratégia dos estoques. Rev Adm [Internet]. 2011; 46(3):290-304. Available from: http://dx.doi.org/10.5700/rausp1013

15. Raimundo EA, Dias CN, Guerra M. Logística de Medicamentos e Materiais em um Hospital público do Distrito Federal-Brasília. Rahis. 2015; 12(2):619.

16. Gazel WF. Indicadores de Desempenho Logístico: Uma Revisão Integrativa da Literatura [dissertação]. 2014. http://bibliotecatede.uninove.br/handle/tede/218

17. Silva JN., Lima JC. Gestão de materiais: almoxarifado hospitalar. Cad Unisuam Pesqui Ext. 2015; 5:35-45.

18. Fernandes, Sheila Mendes et al. Revisão sistemática da literatura sobre as formas de mensuração do desempenho da logística reversa. Gest. Prod. [online]. 2018; 25(1):175-190.

Recebido: 26 de março de 2020. Aceito: 27 de dezembro de 2020

Correspondência: Thais Bento Lima da Silva. E-mail: gerontologathais@gmail.coml 
Conflito de Interesses: os autores declararam não haver conflito de interesses.

(C) This is an Open Access article distributed under the terms of the Creative Commons Attribution License, which permits unrestricted use, distribution, and reproduction in any medium, provided the original work is properly cited 